\section{Die Entstehung des Instituts}

Die Weiterentwicklung der Infektiologie und die Ansiedelung der Tropenmedizin erfolgten unter der Leitung des Direktors der Klinik für Gastroenterologie, Hepatologie und Infektiologie Professor Dr. Dieter Häussinger und bilden heute durch Grundlagenforschung, große klinische Forschungsprojekte und steigende Behandlungszahlen neben der klinischen und experimentellen Hepatologie und Gastroenterologie einen weiteren Schwerpunkt der Klinik, die seit Jahren auch als Zentrum für Infektiologie zertifiziert ist.

Baulich wird diese Entwicklung in der Eröffnung des Leber- und Infektionszentrums im Jahr 2011 widergespiegelt. In diesem hochmodernen Gebäude befindet sich unter anderem auch die einzige Behandlungseinheit in Nordrhein-Westfalen für hochinfektiöse Patienten wie z. B. Ebola-Erkrankte.

Das Streben nach einer Internationalisierung der Infektionsmedizin war ein ambitionierter und konsequenter Schritt. Ziel war die Etablierung einer „Außenstelle“ der Klinik für Gastroenterologie, Hepatologie und Infektiologie in einem tropischen Land mit der Möglichkeit der infektiologischen und tropenmedizinischen Forschung in einem Hochprävalenzland. Weitere Absichten waren der Aufbau einer internationalen Partnerschaft, ständiger bilateraler Wissenstransfer sowie die Unterstützung einer afrikanischen Universität in den Bereichen medizinische Forschung, Lehre und Klinik. Ferner sollte es ermöglicht werden, dass die tropenmedizinische Weiterbildung für Internisten komplett in der Düsseldorfer Klinik unter Leitung von Professor Dr. Häussinger ermöglicht werden kann.

Bei der Suche nach einem geeigneten Standort und einer möglichen längerfristigen universi-

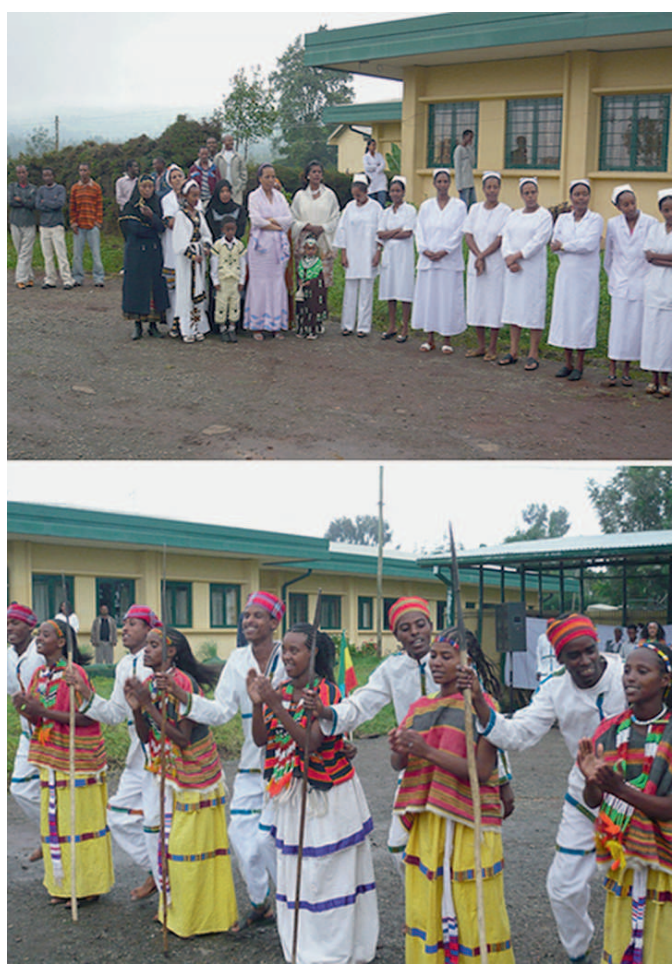

Empfang mit traditionellem Tanz anlässlich des ersten Besuchs von Professor Häussinger im Asella Teaching Hospital im Jahr 2009

tären Partnerschaft boten sich Äthiopien und insbesondere die Universität in Adama an.

In Äthiopien herrscht nach langen Jahren politischer und militärischer Auseinandersetzungen sowie wechselnder repressiver Regime und Dürreperioden mit Hungersnöten eine Phase der politischen Stabilität und des stetigen wirtschaftlichen Aufschwungs. Dennoch ist Äthiopien bis heute eines der ärmsten und am wenigsten entwickelten Länder der Welt. Adama gehört mit etwa 500.000 Einwohnern zu einer Reihe mittelgroßer Städte des Landes. $90 \mathrm{~km}$ nordwestlich befindet sich Addis Abeba, die Hauptstadt und einzige Millionenstadt in Äthiopien. 
Im Jahre 2006 wurde die „Adama Science and Technology University" (ASTU) aus einer Fachschule für Lehrer gegründet. Plan der äthiopischen Regierung war die Etablierung einer Modelluniversität nach deutschem Vorbild. Neben einem deutschen Universitätspräsidenten (Professor Dr. H. Eichele) wurde ein Großteil der Fakultäten von renommierten, erfahrenen Dekanen aus Deutschland geleitet. Wie geplant, lief das Modell der deutschäthiopischen „Doppel-Spitze“ in den Jahren 2011/12 aus. In die rasch florierende Universität waren im Jahr 2013 bis zu 20.000 Studenten eingeschrieben.

Die Medizinische Fakultät der Universität wurde 2009 gegründet. Als Sitz wählte man Asella, eine Kleinstadt, aber regionales Zentrum der von Getreideanbau geprägten Arsi-Zone, $70 \mathrm{~km}$ südlich von Adama. Hier war bereits die Landwirtschaftliche Fakultät angesiedelt.

Das Krankenhaus in Asella ist die höhere Versorgungseinheit für ein Gebiet mit 3,5 Millionen Einwohnern. Aus drei (geplant neun) Distrikt-Krankenhäusern und einer Vielzahl von Gesundheitszentren erfolgt die Zuwei-

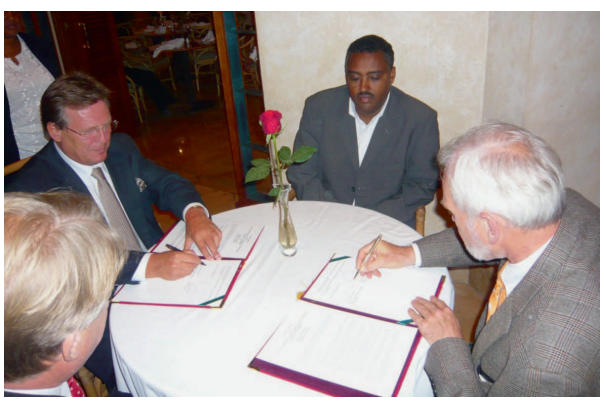

Unterzeichnung eines letter of intent zur künftigen Zusammenarbeit anlässlich eines Besuchs in Äthiopien im September 2009. Herr M. Biontino (Deutscher Vizebotschafter), Professor Dr. D. Häussinger, Seine Exzellenz D. Mekonnen (damaliger Bildungsminister von Äthiopien) und Professor Dr. H. Eichele (damaliger Präsident der Adama Universität) (v.l.n.r.) sung. Zur Verfügung stehen die Fachrichtungen Innere Medizin, Frauenheilkunde, Kinderheilkunde und Allgemeine Chirurgie. Ferner sind eine große HIV/AIDS-Ambulanz, eine augenärztliche Ambulanz, eine zahnmedizinische Ambulanz und ein Projekt für die Versorgung der in Äthiopien häufig vorkommenden rektovaginalen Fisteln dem Krankenhaus angeschlossen.

Dem dortigen Krankenhaus angegliedert, bestand bereits seit vielen Jahren eine Fachschule für Pflegeberufe, Hebammen und Laborassistenten.

Die entstehende Medizinische Fakultät wurde unter dem Dekanat von Herrn Professor Dr. Dietrich Birnbaum (Uniklinik Regensburg, ehem. Ärztlicher Direktor und Geschäftsführer der Kerckhoff-Klinik in Bad Nauheim) maßgeblich aufgebaut und von ihm für mehrere Jahre geleitet.

So bot sich diese junge Medizinische Fakultät als Partner für das Düsseldorfer Projekt zur Etablierung einer langfristigen Partnerschaft besonders gut an, und entsprechende Kontakte wurden etabliert und ausgebaut.

Im September 2009 unterzeichneten Herr Professor Dr. Häussinger, Herr Professor Dr. Eichele und der damalige Bildungsminister Äthiopiens Ato Mekonnen einen entsprechenden „Letter of intent“.

Am 21. Juni 2010 erfolgte in Düsseldorf die Unterzeichnung des Kooperationsvertrags zwischen der Medizinischen Fakultät der Heinrich-Heine-Universität Düsseldorf, dem Universitätsklinikum Düsseldorf und der ASTU.

Nach Planung, Bau und Einrichtung des Instituts erfolgte am 16. Oktober 2013 durch Professor Häussinger, Professor Lee (damaliger Präsident der ASTU), Professor Schnitz- 


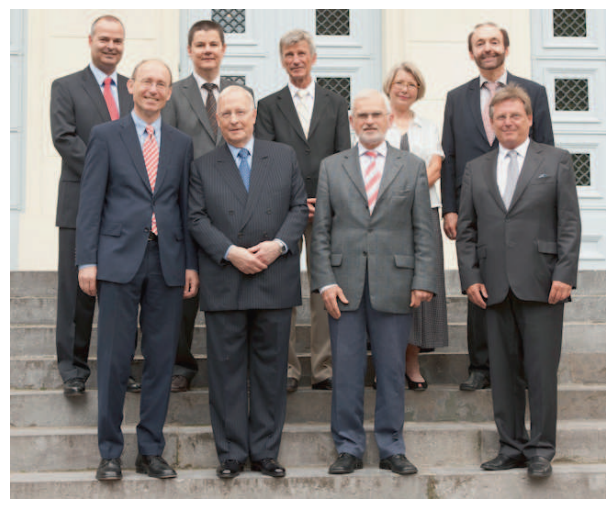

Vertragsunterzeichnung am 21. Juli 2010 zwischen Vertretern der Adama-Universität (Äthiopien), der Heinrich-Heine-Universität (Düsseldorf) und dem Universitätsklinikum Düsseldorf im Schloss Mickeln in Düsseldorf: Vordere Reihe, v.l.n.r.: Professor Dr. Dr. H. M. Piper (Rektor der HHU Düsseldorf), Herr W. Hirsch (Namensgeber und Spender, Gründer und Geschäftsführender Gesellschafter der Hirsch-Gruppe), Professor Dr. H. Eichele (Präsident der Adama University), Professor Dr. D. Häussinger (Direktor der Klinik für Gastroenterologie, Hepatologie und Infektiologie). Hintere Reihe v.l.n.r.: Dr. M. Wokittel (Kaufmännischer Direktor UKD), Professor Dr. J. Windolf (Dekan), Professor Dr. D. E. Birnbaum (ehem. Dekan der Medizinischen Fakultät Adama University), Frau C. Herrmann (Ministerium für Innovation, Wissenschaft und Forschung), Professor Dr. Werner Stüber (Dekan, School of Humanities and Natural Sciences, Adama-Universität)

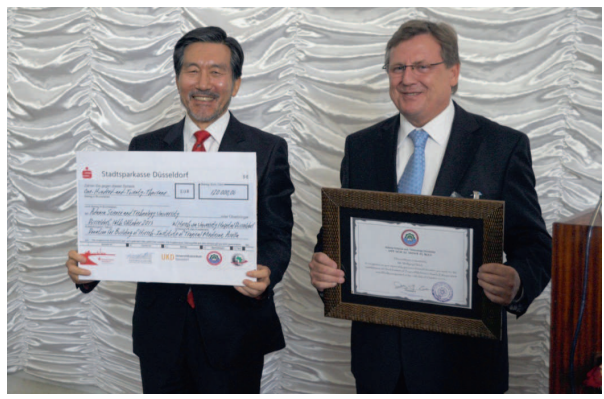

Offizielle Scheckübergabe im Rahmen der Eröffnung im Oktober 2013 durch Professor Häussinger an Professor Lee, Präsident der Adama Science and Technology University

ler (Prorektor der Heinrich-Heine-Universität) und Dr. Legesse (Dekan der Medizinischen Fakultät der ASTU) die feierliche Eröffnung des Instituts. Seit der Eröffnung sind meist zwei ärztliche Mitarbeiter der Klinik für Gastroenterologie, Hepatologie und Infektiologie des Universitätsklinikums Düsseldorf als Koordinatoren des HITM vor Ort in Äthiopien. Aufgrund des kontinuierlichen Einsatzes des Institutsgründers und -leiters, Professor Häussinger, kann seither eine solide finanzielle und logistische Unterstützung durch deutsche Partner, Spender und Organisationen gewährleistet werden.

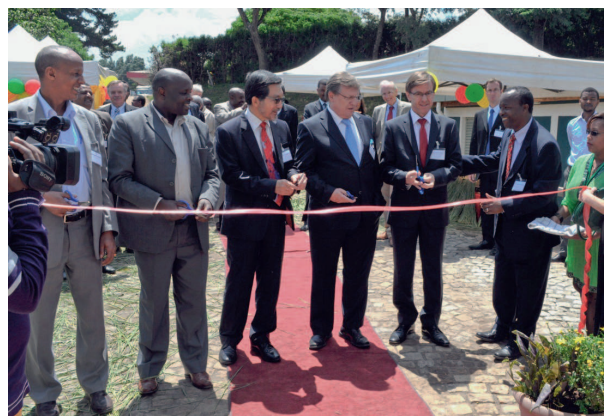

Die Institutseröffnung am 16. Oktober 2013

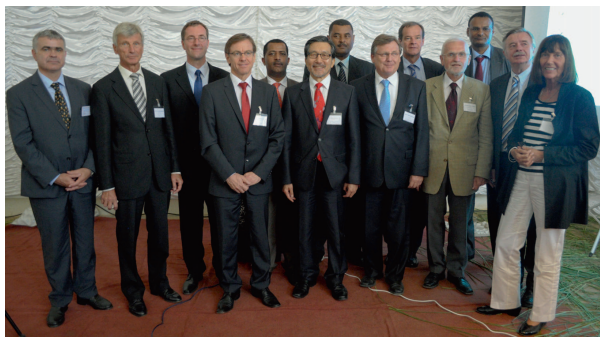

Die Delegationen zur Eröffnung des Instituts: (v.l.n.r.) Professor Gerald Heusing (DAAD), Professor Dietrich Birnbaum (Dekan der Medizinischen Fakultät der ASTU), Dr. Torsten Feldt (Oberarzt und stellvertretender Direktor, HITM), Professor Alfons Schnitzler (Prorektor, HHU), Dr. Lemi Guta (Vizepräsident für Forschung, ASTU), Professor Jang Gyu Lee (Präsident der ASTU), Dr. Habtamu Kabur (Vizepräsident für Verwaltung, ASTU), Professor Dieter Häussinger (Direktor, HITM), Herr Thomas Terstegen (Ständiger Vertreter an der Deutschen Botschaft Addis Abeba), Professor Herbert Eichele (ehem. Präsident der ASTU), Dr. Tolla Beriso (Erster Minister für Bildung und Wissenschaft des Staates Oromia), Professor Herbert Becker (DAAD) und Dr. Irmela Müller-Stöver (Tropenmedizin UKD) 


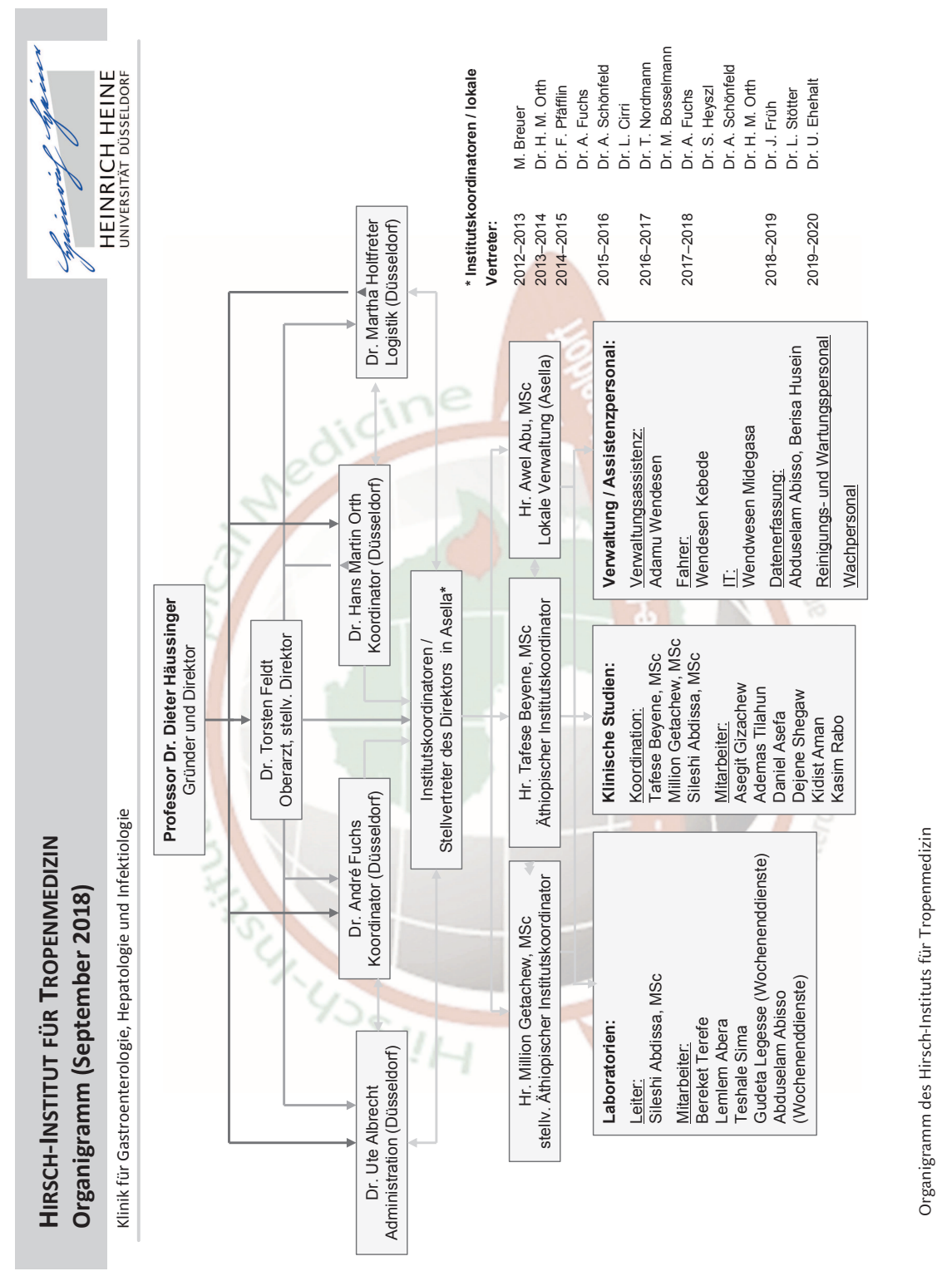




\section{Kaffee-Zeremonie}

Kaffee ist nicht nur eines der wichtigsten Exportgüter Äthiopiens, sondern das Trinken von Kaffee ist auch fest in der äthiopischen Kultur verwurzelt. Zu besonderen Anlässen wird eine Kaffee-Zeremonie durchgeführt, die mit allen darin enthaltenen Schritten deutlich über eine Stunde in Anspruch nehmen kann. Die bewirteten Gäste sitzen auf kleinen Hockern im Halbkreis um diejenige Frau, welche die Kaffeezeremonie ehrenvoll durchführt. Die Zeremonienstätte wird mit frischem Gras auf dem Boden dekoriert. Ein Feuer wird entfacht. Mit einem Stück Glut entzündet die Zeremonien-Leiterin Weihrauch, welcher einen wohlriechenden Duft im Raum verbreitet. Auf der Glut werden traditionell Kichererbsen oder Weizen geräuchert, um später zum Kaffee als Snack gereicht zu werden.

Anschließend werden grüne Kaffeebohnen gewaschen und in einer Schale über der Glut geröstet. Aufsteigender Kaffeeduft verheißt nahenden Genuss. Nun wird das Wasser in einer Tonkanne erhitzt, während die gerösteten Bohnen in einem hölzernen Mörser zu Pulver zerstoßen werden. Das so gewonnene, frisch geröstete und gemahlene Kaffeepulver wird dem kochenden Wasser hinzugegeben. Nach wenigen Minuten ist der Kaffee fertig und wird in kleinen schalenförmigen Tassen serviert. Die als erste befüllte Tasse wird für „Mutter Erde“ ausgeschenkt und nicht getrunken. Der geübten Frau gelingt es, alle Tassen in einem einzigen Ausgieß-Vorgang zu füllen. Dem traditionell zubereiteten, sehr starken Kaffee wird vor dem Trinken viel Zucker zugemischt. Während die Gäste der aufwändigen Zeremonie beiwohnen, findet eine rege Unterhaltung aller Beteiligten statt. Die Kaffee-Zeremonie wird beispielsweise abgehalten, wenn die deutschen Mitarbeiter des Instituts in Asella zu den Familien der äthiopischen Kollegen und Mitarbeiter eingeladen werden, oder besonders aufwändig, wenn ein deutscher Mitarbeiter seinen Einsatz in Asella beendet. Neben dem Kaffeegenuss erfüllt die eindrucksvolle Zeremonie aber auch einen wichtigen kommunikativen Zweck: In Äthiopien kann während einer Tasse Kaffee über alles offen geredet werden. 


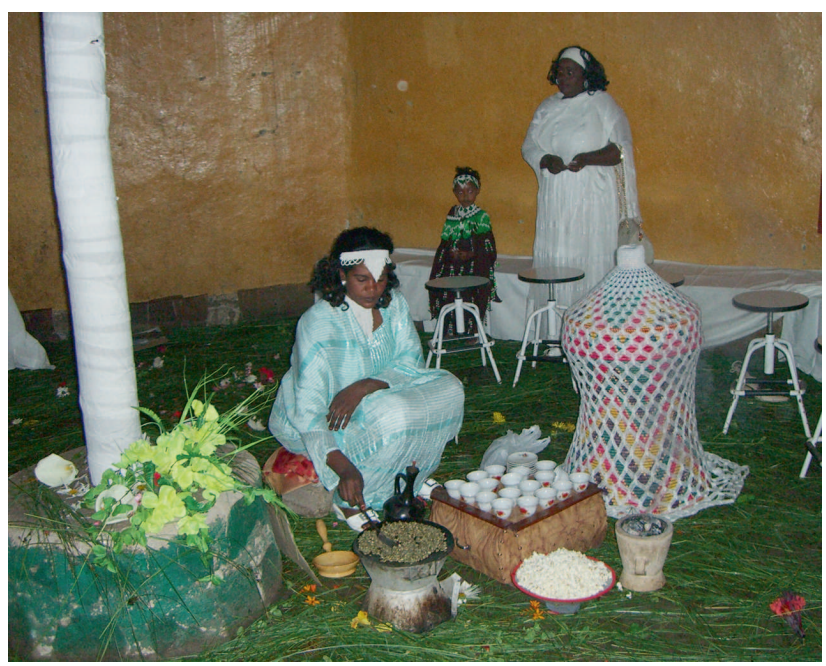

Traditionelle Kaffee-Zeremonie

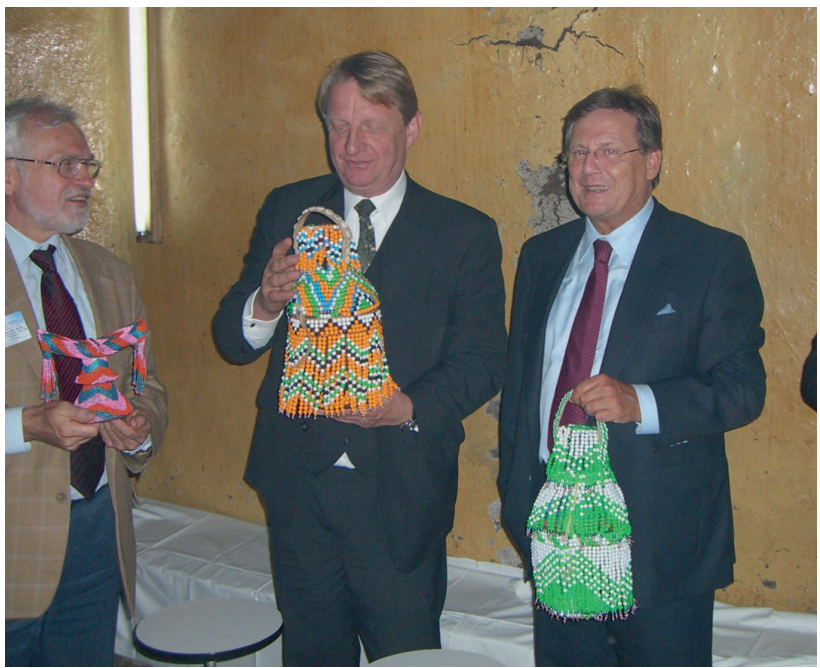

Im Anschluss an die Zeremonie werden Geschenke überreicht 